Revue de droit comparé du travail et de la sécurité sociale

$2 \mid 2020$

La vie personnelle du salarié

\title{
L'utilisation des réseaux sociaux par les salariés en Australie
}

Gabrielle Golding

\section{(2) OpenEdition}

12 Journals

Édition électronique

URL : https://journals.openedition.org/rdctss/1052

DOI : $10.4000 /$ rdctss. 1052

ISSN : 2262-9815

Éditeur

Centre de droit comparé du travail et de la sécurité sociale

Édition imprimée

Date de publication : 1 juin 2020

Pagination : 24-35

ISSN : 2117-4350

\section{Référence électronique}

Gabrielle Golding, "L'utilisation des réseaux sociaux par les salariés en Australie », Revue de droit comparé du travail et de la sécurité sociale [En ligne], 2 | 2020, mis en ligne le 01 novembre 2021, consulté le 11 novembre 2021. URL : http://journals.openedition.org/rdctss/1052 ; DOI : https:// doi.org/10.4000/rdctss. 1052

\section{(c) (†) $९$}

Revue de droit comparé du travail et de la sécurité sociale est mise à disposition selon les termes de la Licence Creative Commons Attribution - Pas d'Utilisation Commerciale - Pas de Modification 4.0 International. 


\section{L'UTILISATION DES RÉSEAUX SOCIAUX PAR LES SALARIÉS EN AUSTRALIE}

$\$$

\section{GABRIELLE GOLDING}

Maître de conférences, Université d'Adélaïde, membre du groupe de recherche sur la réglementation du travail et de l'emploi, et du groupe de recherche en

droit commercial de la faculté de droit d'Adélaïde

\section{RÉSUMÉ}

Cet article fait le point sur la récente jurisprudence australienne concernant le licenciement d'employés en raison de leur comportement en dehors des heures d'ouverture sur les réseaux sociaux. Il examine également la justification d'une éventuelle modification de la loi régissant ces licenciements en Australie.

Mots-clés : Comportement en dehors des heures d'ouverture, médias sociaux, licenciement, action défavorable, Droit du travail australien

\section{ABSTRACT}

This article provides an update on recent Australian case law concerning the dismissal of employees by reason of their out-of-hours conduct on social media. It also considers the rationale for potential change to the law governing such dismissals in Australia.

Key words: Out-of-hours Conduct, Social Media, Dismissal, Adverse Action, Australian employment law 


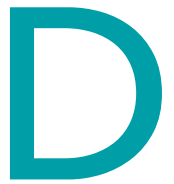

ans une société constamment connectée, où l'Internet joue un rôle de premier plan, il n'est pas étonnant que l'utilisation des réseaux sociaux par les salariés en Australie ait pu poser des problèmes à leurs employeurs, d'autant plus lorsque cette activité peut motiver un licenciement. La présente étude présente une analyse de la jurisprudence récente sur l'utilisation des réseaux sociaux par les salariés, en dehors des heures de travail, et du licenciement susceptible d'en découler. Cet article s'intéresse aussi aux arguments exprimés en faveur d'une modification de la loi qui régit actuellement ces licenciements.

L'utilisation quotidienne des réseaux sociaux a pris une ampleur suffisante pour que I'on s'interroge : un employeur en Australie a-t-il le droit de licencier un salarié au regard de ses dires ou agissements sur les réseaux sociaux, même si ceux-ci ont eu lieu durant son temps libre? La réponse à cette question est tout simplement positive notamment si l'employeur peut prouver que les actions de ce salarié nuisent à l'image de l'entreprise (I) ${ }^{1}$. Les licenciements liés au comportement sur les réseaux sociaux en dehors des heures de travail sont réglementés en Australie, à la fois par la loi comme par les politiques et les Codes de conduite mis en place par les employeurs en la matière.

Malgré cette apparente simplicité, les récents développements judiciaires rendent la réponse à cette question un peu plus complexe, selon la nature du contenu qu'un salarié choisit de communiquer sur les réseaux sociaux (II). Ces décisions judiciaires et l'analyse de la jurisprudence des 12 derniers mois montrent que les réseaux sociaux font désormais partie intégrante de la vie quotidienne en Australie, les employeurs, la loi et les tribunaux du travail essayant constamment de s'adapter à cette évolution.

Plus important encore peut-être, et alors que l'Australie ne dispose pas de Déclaration des droits de l'homme, la Haute Cour australienne a récemment découvert que la liberté de communication politique est implicite dans la Constitution australienne, mais ce n'est pas un droit individuel. Si la Constitution protège la communication politique, c'est pour défendre les intérêts de l'Etat, dans son ensemble². La décision rendue dans l'affaire Comcare v. Banerji en constitue un bon exemple ${ }^{3}$.

Plusieurs recommandations peuvent être formulées afin de modifier la loi australienne concernant le licenciement motivé par l'utilisation des réseaux sociaux en dehors des heures de travail. La principale recommandation consiste à établir des limites plus claires quant au pouvoir de l'employeur, par exemple via une politique ou un Code de conduite en matière de réseaux sociaux.

1 Voir, par exemple, Rose v Telstra Corporation [1998] AIRC 1592 (Rose), [30].

2 [2019] HCA 23, [20].

3 [2019] HCA 23 (Banerji). 
Certes, il existe déjà des études relatives au licenciement abusif et à la conduite des salariés sur les réseaux sociaux en dehors des heures de travail4. Toutefois, il ne semble pas y avoir d'analyse jurisprudentielle récente des décisions étudiées dans cet article, alors que ces développements pourraient délimiter plus clairement le contrôle exercé par l'employeur en matière de réseaux sociaux.

\section{I - LA RÉGLEMENTATION DU COMPORTEMENT SUR LES RÉSEAUX SOCIAUX EN DEHORS DES HEURES DE TRAVAIL}

Il existe un certain nombre de voies permettant à un salarié en Australie de demander réparation en cas de licenciement abusif lié à son comportement sur les réseaux sociaux en dehors du travail en faisant appel au juge (A) ou en s'appuyant sur les politiques ou codes de conduite des entreprises (B).

\section{A - LES RECOURS JURIDIQUES}

Le droit du travail australien exige qu'un salarié coopère avec son employeur ${ }^{5}$ et ne se livre à aucun comportement susceptible de nuire aux activités ou à l'image de son entreprise ${ }^{6}$. II s'agit là de conditions fondamentales de droit commun, comprises implicitement dans le contrat de travail de tout salarié ${ }^{7}$. De plus en plus souvent, les tribunaux australiens du travail en sont venus à interpréter ces principes de manière à permettre aux employeurs de contrôler le comportement des salariés en dehors des heures de travail ${ }^{8}$.

Sur la base de ces conditions implicites, le licenciement d'un salarié pourrait être légal s'il publiait sur Facebook des commentaires désobligeants concernant son employeur, à condition que celui-ci puisse prouver qu'il existe un «lien suffisant » avec son emploi ${ }^{9}$. Ce lien existerait même dans le cas où, par exemple, une publication apparemment " privée " sur Facebook nuirait à la réputation de l'employeur ${ }^{10}$.

Ainsi, Fair Work Australia - désormais appelée Fair Work Commission (Commission du travail équitable $)^{11}$ - a validé le licenciement d'un salarié en raison de commentaires publiés sur Facebook à propos de ses collègues, l'une de ses remarques pouvant même être interprétée comme une menace ${ }^{12}$.

4 Voir L. Thornwaite, "Social Media and Dismissal: Towards a Reasonable Expectation of Privacy? », 2018, n60, JIR, p. 119; L. Thornwaite, "Social Media, Unfair Dismissal and the Regulation of Employees », Conduct Outside Work', 2013, n²6, AJL, p. 164.

5 Voir Commonwealth Bank of Australia v. Barker (2014) 253 CLR 169, [25]-[27].

6 Voir R W Jaksch \& Associates Pty Ltd v. Hawks [2005] VSCA 307, [60]-[72].

7 Ces conditions sont implicites dans la classe des contrats de travail, tant qu'elles sont nécessaires : voir G. Golding, «Terms Implied by Law Into Employment Contracts: Are they Necessary? », 2015, $n^{\circ} 28, A J L L$, p. 113.

8 Voir A. Stewart, Stewart's Guide to Employment Law, 5e éd., Federation Press, 2015, p. 261.

9 Voir par exemple Rose, [30].

10 Voir Fitzgerald v. Smith t/as Escape Hair Design [2010] FWA 7358, [50].

11 Voir Explanatory Memorandum, Fair Work Amendment Bill 2012 (Cth), p.1 et 9.

12 Voir O'Keefe v. Williams Muir's Pty Limited T/A Troy Williams The Good Guys [2011] FWA 5311, [42][43]. 
Pourtant, la Commission a jugé, dans une autre affaire, qu'un employeur avait injustement licencié un salarié ayant tenu des commentaires "grossiers et vulgaires » sur Facebook, car lesdites remarques concernaient l'entreprise où travaillait sa mère, et non la sienne ${ }^{13}$. II n'y avait donc pas de « lien suffisant » avec son emploi.

Toute plainte pour licenciement abusif suppose que la Commission examine au cas par cas les agissements du salarié et détermine si, dans l'ensemble, il a été traité équitablement en termes de procédure et de règles substantielles ${ }^{14}$. Si ce salarié obtient gain de cause dans une plainte pour licenciement abusif, il pourra recevoir jusqu'à 6 mois de salaire à titre $d^{\prime}$ indemnisation ${ }^{15}$. La réintégration est théoriquement le mode de réparation " privilégié »" mais elle est rarement accordée puisque, le plus fréquemment, la relation entre l'employeur et le salarié a été brisée de façon irrémédiable ${ }^{17}$.

Lorsqu'un salarié est licencié pour avoir exprimé une opinion politique sur les réseaux sociaux, il peut par ailleurs intenter une action en justice en vertu de la loi sur le travail équitable ${ }^{18}$. Néanmoins, une telle action ne peut être engagée simultanément avec une plainte pour licenciement abusif, le salarié peut envisager l'une ou l'autre, mais pas les deux ${ }^{19}$. S'il n'existe pas de limite au montant de l'indemnité pouvant être octroyée en réparation des dommages subis, en revanche un salarié doit toujours prouver le préjudice dont il a souffert ${ }^{20}$.

Par ailleurs, il doit apporter la preuve que son employeur a pris des mesures qui lui étaient préjudiciables en mettant fin à son emploi, et que son licenciement était en réalité motivé par ses opinions politiques ${ }^{21}$. S'il peut le démontrer, la charge de la preuve est renversée et il incombe alors à l'employeur d'établir que les opinions politiques du salarié n'ont joué aucun rôle dans la décision de le licencier ${ }^{22}$.

En cas de procédure pour licenciement abusif, un employeur peut essayer de contester cette accusation en faisant valoir que le seul motif du licenciement était la violation d'une politique de l'entreprise, et non le contenu d'une activité du salarié sur les réseaux sociaux ${ }^{23}$.

Dans ce contexte, on peut imaginer qu'il devrait y avoir une limite à l'étendue du pouvoir de contrôle exercé par l'employeur ou par le biais d'une politique d'entreprise, sur

13 Voir Somogyi v LED Technologies Pty Ltd [2017] FWC 1966, [39].

14 Fair Work Act 2009 (Cth) (FW Act), p. 385. Sur les critères d'éligibilité d'une telle demande, voir Stewart, $n^{\circ} 8$, p. 366. II importe de noter qu'il existe toute une gamme de possibilités offertes aux salariés pour contester leur licenciement en vertu de la législation nationale et fédérale et de la Common Law. Voir par exemple Stewart, $n^{\circ} 8$, p. 360.

15 Ibid., p. 392(1).

16 Ibid., p. 391(1).

17 Voir E. Shi et F.N. Zhong, Rethinking the Reinstatement Remedy in Unfair Dismissal Law, 2018, 39, Adel L R, p. 363.

18 FW Act s 351(1).

19 Ibid., p. 725.

20 Ibid., p. 550(1).

21 Ibid., p. 361(1).

22 Ibid., p. 351(1)

23 Voir Browne v. Coles Group Supply Chain Pty Ltd [2014] FWC 3670, [62]. Un salarié peut également prétendre avoir été victime de discrimination en vertu d'une loi anti-discrimination d'un Etat/ Territoire. 
des contenus qui ne sont pas liés à l'entreprise. Comme analysé ci-avant, cette question n'est pas précisément tranchée par le droit australien et il n'existe pas encore de décision judiciaire définitive à ce sujet.

\section{B - Politiques internes et CODEs de CONDUite}

Les employeurs cherchent souvent à étendre les obligations des salariés en vertu du droit commun. A cet effet, ils élaborent des politiques internes et/ou des codes de conduite, qui réglementent généralement les comportements à adopter sur les réseaux sociaux. L'employeur est alors en mesure de sanctionner ou de licencier un salarié pour comportement inapproprié, même en dehors du lieu ou des heures de travail, en particulier lorsque celui-ci a signé un contrat dans lequel il s'engage à respecter ces politiques ${ }^{24}$.

Ainsi, les employeurs sont autorisés à sanctionner un salarié en raison d'une utilisation inappropriée des réseaux sociaux, à condition d'avoir expliqué la politique interne en vigueur et d'avoir dispensé une formation à ce sujet, sous quelque forme que ce soit ${ }^{25}$. Néanmoins, un certain nombre de salariés ont intenté avec succès des procédures pour licenciement abusif devant la Commission, affirmant qu'ils n'avaient pas compris que leurs publications pouvaient être vues par le public ${ }^{26}$ ou qu'il était possible de les associer à leur entreprise $^{27}$.

A plusieurs reprises, les tribunaux ont jugé qu'une violation d'une politique interne relative aux réseaux sociaux pouvait justifier un licenciement, mais les employeurs ne sont toutefois pas autorisés à appliquer une politique qui s'étende aux affaires privées, sans lien avec le lieu de travail ou les affaires de l'employeur ${ }^{28}$.

\section{II - LES DÉVELOPPEMENTS RÉCENTS}

Sont ici examinées quatre décisions sur des procédures de licenciement abusif datant des douze derniers mois, dans lesquelles les salariés contestaient un licenciement motivé par leur comportement sur les réseaux sociaux en dehors des heures de travail.

Dans tous les cas sauf un, le licenciement a été validé au motif que le comportement de ces salariés présentait un lien suffisant avec leur emploi. Dans chaque cas, l'employeur avait mis en place une politique interne et les salariés n'ont pas réussi à contester la portée et les limites de cette politique. Néanmoins, et comme cela a été suggéré ci-dessus, il est possible que cela se produise à l'avenir.

24 Voir Chittick v. Ackland (1984) 53 ALR 143.

25 Voir Romero v. Farstad Shipping (Indian Pacific) Pty Ltd (2014) 231 FCR 403, [60].

26 Voir Linfox Australia Pty Ltd v. Glen Stutsel [2012] FWAFB 7097.

27 Voir Dover-Ray v. Real Insurance Pty Ltd [2010] FWA 8544.

28 Voir Kolodjashnij v. Lion Nathan T/A J Boag and Son Brewing Pty Ltd [2009] AIRC 893, [52]. 


\section{A - L'Affaire Tracey V. BP Refinery (KWINANA) PTy LTD ${ }^{29}$}

Dans l'affaire Tracey, un technicien a été renvoyé sans préavis en janvier 2019 après avoir publié une vidéo intitulée « Hitler Parody EA Negotiations » (parodie d'Hitler dans la négociation des accords d'entreprise), accessible par un groupe Facebook fermé réservé aux salariés.

Cette vidéo consistait en un montage, réalisé à partir de «La Chute » - un film allemand sorti en 2004 - consacré aux derniers jours de la vie d'Hitler, qui mettait en scène les négociations entre les représentants de salariés et les dirigeants de l'entreprise.

En validant le licenciement du technicien, la vice-présidente Binet a rejeté l'argument du salarié selon lequel la vidéo « humoristique » visait à « remonter le moral » des collègues au moment où, après 16 mois de négociations d'entreprise, l'employeur demandait la résiliation d'un accord ayant expiré.

La Vice-présidente Binet n'a pas jugé que la création et le partage de la vidéo avec d'autres salariés puissent être considérés comme " une plaisanterie » ou comme "une façon de dédramatiser " d'interminables négociations, relevant qu'il était difficile de concevoir que le technicien puisse ne pas comprendre que cette vidéo était « insultante et inappropriée ». La Vice-présidente a estimé que le simple fait de "cataloguer quelque chose comme une parodie n'excusait pas tout et n'importe quoi » et ne signifiait pas nécessairement que ce n'était "pas insultant $»^{30}$.

Au cours des échanges, l'employeur a déclaré à la Commission que «la vidéo semblait dépeindre un certain nombre de [ses] salariés comme des nazis et faisait référence à des informations très précises concernant ce qui se passait (...) à l'époque et aux négociations en cours (...) sur les accords d'entreprise ». La vidéo contenait notamment des phrases " utilisées par les salariés tout au long du processus de négociation » et faisait référence à une «série de présentations » effectuées durant les négociations.

Le technicien a reconnu avoir partagé la vidéo sur Facebook avec un groupe de collègues et avec d'autres collègues au sein de l'entreprise, affirmant néanmoins que son contenu avait été "créé par son épouse, de sa propre initiative ». Toutefois, la Vice-présidente a rejeté le témoignage de l'épouse du salarié à l'appui de cette affirmation, estimant « [qu'elle] n'était pas en mesure d'expliquer bon nombre des paroles et concepts de la vidéo qu'elle prétendait avoir créée $»^{31}$.

La Vice-présidente a exprimé son accord avec l'employeur sur le fait qu'en synchronisant le discours d'Hitler avec les mots prononcés par ses cadres dirigeants, " la vidéo suggère une similitude $»^{32}$.

29 [2019] FWC 4113.

30 Ibid., [99].

31 Ibid., [13].

32 Ibid., [84]. 
Par ailleurs, elle s'est appuyée sur des décisions antérieures dans lesquelles un salarié avait "fait référence, ou comparé son employeur, à Hitler ou au régime nazi » ${ }^{33}$, ainsi convaincue que toute personne raisonnable jugerait la vidéo sur Hitler inappropriée, insultante, et contraire aux politiques en vigueur dans l'entreprise ${ }^{34}$.

Concluant que le licenciement du technicien était dûment motivé, la vice-présidente a estimé que sa conduite démontrait une «brèche affectant les fondements de sa relation de confiance et de respect avec son employeur ${ }^{35}$. Bien que le technicien ait exprimé des remords, "étant donné son manque de discernement ", il était donc " probable qu'il entrerait de nouveau en conflit avec son employeur ».

Finalement «compte tenu du lourd impact émotionnel et financier du licenciement sur [le technicien] et sa famille, et [eu égard au] paiement d'une indemnité tenant lieu de préavis (...) son licenciement n'était ni trop sévère, ni injuste, ni déraisonnable $»^{36}$.

\section{B - L'Affaire Murkitt v. Staysafe Security T/A Alarmnet Monitoring ${ }^{37}$}

Contrairement à l'affaire Tracey, la Commission a jugé dans l'affaire Murkitt que l'entreprise Alarmnet avait réagi de manière excessive en congédiant une salariée de longue date pour une violation isolée de sa politique en matière de réseaux sociaux, alors qu'elle avait publié des commentaires désobligeants sur Facebook concernant les administrateurs de l'entreprise.

Selon les témoignages entendus par le Commissaire Platt, cette ancienne opératrice de salle de contrôle travaillait pour Alarmnet depuis près de 15 ans lorsqu'elle s'est exprimée sur Facebook, en février 2019, exprimant sa frustration à l'égard des nouveaux propriétaires de l'entreprise. Dans sa publication, elle décrivait son poste comme " ingrat ", regrettant que les administrateurs "ne se soucient ni des clients, [ni] de leur personnel», exprimant sa tristesse en ces termes : "Mes chers Adélaïdediens (sic), j'adore Adélaïde. Ces trois Victoriens sont arrivés et ont tout changé $»^{38}$.

La salariée, qui percevait des indemnités pour accident du travail lorsqu'elle a publié ces commentaires, a témoigné qu'elle souffrait d'une blessure psychologique attribuée, selon des certificats médicaux, à l'attitude de la direction de son entreprise. En outre, elle a déclaré à la Commission qu'elle était bouleversée par la manière dont les nouveaux administrateurs avaient géré le décès récent d'un salarié et par le fait qu'ils n'avaient pas suffisamment soutenu les travailleurs après l'incident ${ }^{39}$.

\footnotetext{
33 Ibid., [101]-[104].

34 Ibid., [105].

35 Ibid., [200].

36 Ibid., [207]-[208].

37 [2019] FWC 5622.

38 Ibid., [25].

39 Ibid., [55].
} 
Pour sa part, Alarmnet a affirmé avoir tenu compte de l'état de santé de la salariée lors de l'examen de sa faute, mais que dans la mesure où elle n'assumait pas la responsabilité de ses actes, son licenciement sans préavis était le résultat approprié. La société a déclaré que la salariée était informée de sa politique en matière de réseaux sociaux, que sa conduite était délibérée et qu'elle n'exprimait aucun remord.

De plus, cette publication sur Facebook attaquait l'entreprise, ses directeurs et le service qu'elle proposait. Enfin, la salariée avait perdu la faveur de son employeur dès lors qu'elle a envoyé un message signifiant son intention de «régler les choses " avec le membre du personnel ayant « divulgué » ses commentaires aux " patrons ${ }^{40}$.

La Commission a également entendu des témoignages selon lesquels elle avait adressé au chef d'entreprise d'Alarmnet, et à d'autres salariés, « un grand nombre » de courriels de " harcèlement et d'intimidation » après son licenciement ${ }^{41}$. D'après la salariée en revanche, sa publication sur Facebook n'avait pas affecté financièrement Alarmnet et elle considérait avoir été licenciée en raison de sa demande d'indemnités pour accident du travail ${ }^{42}$.

Au final, le Commissaire Platt a conclu que le licenciement de la salariée était une réponse disproportionnée au regard de son état de santé préexistant, estimant toutefois que les preuves visant à étayer la thèse d'un licenciement pour éviter de verser les indemnités d'accident du travail étaient insuffisantes, et que la conduite de la salariée constituait effectivement un motif valable de licenciement ${ }^{43}$.

Cependant, l'employeur avait traité trop durement la faute de la salariée dans la mesure où « la sanction du licenciement, à la lumière de [son] état de santé, de son ancienneté, de l'absence de problème dans ses performances jusque-là, était hors de proportion avec la faute $»^{44}$. Le Commissaire a poursuivi en déclarant que si l'état de santé de la salariée n'excusait pas sa conduite, il l'expliquait « dans une certaine mesure ». II a ainsi tenu compte du fait que cette publication était « un événement isolé » et «n'avait causé aucun préjudice financier » à Alarmnet, mais il a admis que cela causait une «perturbation » sur le lieu de travail $^{45}$.

Lors de l'évaluation de l'indemnisation, la Commissaire Platt a estimé que le versement d'une indemnisation ne s'imposait pas car, en tout état de cause, la salariée « n'aurait pas continué à travailler pour Alarmnet si elle n'avait pas été licenciée». Elle percevait également des indemnités pour accident du travail et avait commis une faute ${ }^{46}$.

\footnotetext{
40 Ibid., [25].

41 Ibid., [26].

42 Ibid., [20].

43 Ibid., [44].

44 Ibid., [55].

45 Ibid.

46 Ibid., [80].
} 


\section{C - L'AFFAIRE BANERJI}

Dans l'affaire Banerji, la Haute Cour a déclaré fondé un appel contre un jugement selon lequel le ministère de l'immigration et de la citoyenneté avait abusivement licencié une salariée qui avait publié plus de 9000 tweets via le compte Twitter «@LaLegale » ${ }^{47}$.

En 2013, le ministère avait licencié Mme Banerji pour des tweets anonymes critiquant le gouvernement australien, les politiques d'immigration de l'opposition, les parlementaires, ainsi que son supérieur hiérarchique direct, et dénonçant également son échec à l'obtention d'un emploi extérieur en tant que psychanalyste.

Dans une décision de première instance rendue en août 2014, un agent de Comcare a rejeté sa demande d'indemnisation motivée par un état psychologique susceptible d'être aggravé par le licenciement, jugeant que le préjudice n'était pas constitué dans la mesure où le ministère avait pris à son encontre des mesures administratives raisonnables, appliquées de manière raisonnable ${ }^{48}$.

Mme Banerji a alors interjeté appel devant le Tribunal d'appel administratif (AAT) qui a jugé que son licenciement empiétait sur ses droits constitutionnels implicites, qu'il ne répondait pas au critère des "mesures administratives raisonnables » et qu'il présentait "une ressemblance désagréable avec le crime de pensée de George Orwell » ${ }^{49}$. L'AAT a conclu que, la loi sur la fonction publique de 1999 (Cth) imposant une restriction injustifiée à la liberté implicite de communication politique, son licenciement pour violation du code de conduite de la fonction publique australienne (APS) n'était pas une action administrative raisonnable, menée de manière raisonnable ${ }^{50}$.

Cependant, la décision de l'AAT a été infirmée par la Haute Cour qui a confirmé la décision de 2014 du responsable de Comcare, jugeant que « les dispositions contestées [de la loi] n'imposaient pas une restriction injustifiée à la liberté implicite de communication politique » et que le licenciement de Mme Banerji était légitime ${ }^{51}$.

Dans leur jugement conjoint, le juge en chef Kiefel, les juges Bell, Keane et Nettle ont estimé qu'il était " hautement souhaitable, sinon essentiel au bon fonctionnement d'un système de gouvernement représentatif et responsable", que les gouvernements de tous bords « aient confiance en la capacité de la fonction publique australienne de fournir des conseils professionnels d'une qualité et d'une impartialité parfaites $\|^{52}$. En outre, ils ont jugé que les gouvernements devaient également pouvoir compter sur une "fonction publique australienne [mettant] fidèlement et professionnellement en œuvre la politique gouvernementale, quelles que soient les convictions et les choix politiques personnels des fonctionnaires ${ }^{53}$.

\footnotetext{
47 Banerji, [2].

48 Ibid., [11].

49 Banerji and Comcare (Compensation) [2018] AATA 892, [116].

50 Ibid., [128].

51 Banerji, [1].

52 lbid., [34].

53 Ibid.
} 


\section{AUSTRALIE}

Par ailleurs, la liberté implicite de communication politique n'apparaissait pas comme « un droit personnel à la liberté d'expression », mais plutôt comme une restriction du pouvoir législatif susceptible de s'étendre uniquement " pour préserver et protéger un système de gouvernement représentatif et responsable ${ }^{54}$.

S'agissant de l'argument de Mme Banerji selon lequel ces dispositions ne devraient pas concerner les communications « anonymes », les juges Kiefel, Bell, Keane et Nettle ont estimé qu'il « devait être rejeté », étant donné le risque qu'elles « cessent d'être anonymes et portent alors atteinte à l'intégrité et à la bonne réputation de l'APS en tant que service public apolitique et professionnel $»^{55}$. La majorité a également jugé qu'une telle communication " peut nuire à la bonne réputation de l'APS même si elle reste anonyme ${ }^{56}$.

En conclusion, la Haute Cour a infirmé la décision de I'AAT et confirmé la décision de 2014 de Comcare, condamnant Mme Banerji aux dépens au profit de Comcare ${ }^{57}$. En rendant ces ordonnances, le tribunal a été convaincu que les dispositions du code de conduite de la fonction publique australienne étaient raisonnablement appropriées et proportionnées, et n'imposaient pas une restriction injustifiée à la liberté implicite de communication politique ${ }^{58}$.

\section{D - L'AFFAIRE FUSSELL V. TRANSPORT FOR NSW T/A TRANSPORT FOR NSW ${ }^{59}$}

L'affaire Fussell concernait la légitimité du licenciement d'un salarié qui affirmait avoir accidentellement envoyé une photo de son pénis en érection à une collègue via, en dehors des heures de travail. En effet, cet agent de sécurité ferroviaire a déclaré qu'il avait l'intention d'envoyer à sa collègue l'image d'un nouveau tatouage sur son bras, mais avait commis une « erreur de bonne foi » en lui envoyant l'image incriminée ${ }^{60}$.

Le salarié a déclaré au Vice-président Bull de la Commission qu'il s'était immédiatement excusé d'avoir envoyé l'image en question, mais que sa collègue l'avait conservée en prenant une "capture d'écran » et en la transmettant à d'autres salariés, exprimant son dégoût devant son comportement.

Elle s'était également plainte auprès d'un supérieur, en déclarant ne l'avoir " ni demandé, ni souhaité ${ }^{61}$. La salariée a également fait part aux enquêteurs de son inquiétude concernant les contacts à venir avec son collègue, lorsqu'elle reprendrait son travail au terme de son congé de maternitéb2.

\footnotetext{
54 Ibid., [20].

55 Ibid., [36].

56 lbid.

57 Ibid., [47].

58 Ibid., [41].

59 [2019] FWC 1182.

60 Ibid., [16].

61 Ibid., [61].

62 Ibid., [109].
} 
Après avoir pris connaissance de l'image, l'employeur a suspendu l'agent le temps qu'il mène une enquête, puis l'a licencié pour avoir enfreint le code de conduite et la politique interne de l'entreprise en matière d'intimidation, de harcèlement et de réseaux sociaux ${ }^{63}$. L'agent de sécurité a expliqué en partie la présence de cette image sur son téléphone grâce à une lettre de sa nouvelle partenaire, affirmant qu'ils " avaient une relation très visuelle et partageaient souvent des images à caractère personnel et privé ${ }^{64}$.

Néanmoins, le Vice-président Bull a conclu que l'agent avait délibérément envoyé l'image et que ses tentatives pour prétendre le contraire " exigeraient une crédulité sortant de l'ordinaire ${ }^{65}$. L'envoi de cette image « avait peut-être été une erreur ponctuelle », mais "d'une grossièreté aussi extrême qu'il est possible de l'être " ${ }^{66}$. Le Vice-président a jugé que ce comportement en dehors des heures de travail était en rapport avec l'emploi de l'agent puisqu'il enfreignait les politiques internes de l'employeur en matière de réseaux sociaux, s'appliquant à toutes les interactions des salariés avec leurs collègues ${ }^{67}$. II a déclaré qu'il trouvait que le comportement de l'agent justifiait une rupture de la relation ${ }^{68}$; l'envoi de l'image enfreignait les politiques de l'employeur et constituait un motif valable de licenciement ${ }^{69}$.

Si le Vice-président Bull a admis que l'employeur avait «malheureusement et de manière surprenante " utilisé une procédure inappropriée dans la présentation de ses conclusions à l'agent de sécurité, celui-ci avait finalement eu la possibilité de présenter l'intégralité de son dossier, à la fois au sein de l'organisation et devant la Commission ${ }^{70}$. Par conséquent, son licenciement était légitime et n'était ni excessivement sévère, ni injuste, ni déraisonnable ${ }^{71}$.

\section{Conclusion}

Ces affaires démontrent qu'il existe une tension continue entre le droit à au respect ce la vie privée du salarié et l'utilisation des médias sociaux qui peut - ou non - être considérée comme en lien avec son emploi.

Compte tenu de la tension illustrée dans ces affaires, il serait assurément indispensable que des limites soient apportées au pouvoir de l'employeur de restreindre les droits fondamentaux des salariés, dès lors que ceux-ci sont exercés sans aucun lien manifeste avec les affaires de l'employeur ou avec l'entreprise.

\footnotetext{
63 Ibid., [78].

64 Ibid., [22].

65 Ibid., [101].

66 Ibid., [135].

67 Ibid., [108].

68 Ibid., [110].

69 Ibid.

70 Ibid., [120].

71 Ibid., [137].
} 
II ressort en particulier des conclusions de la Haute Cour dans l'affaire Banerji, qu'un employeur ne devrait pas être en mesure d'empêcher ses salariés de faire du lobbying, ou de critiquer les politiques ou les lois gouvernementales sans rapport avec son travail.

Etant donné qu'en Australie, il n'existe pas de Déclaration des droits de la personne pour protéger le droit individuel à la liberté d'expression, et qu'aucune disposition législative spécifique ne protège le droit d'un individu d'exprimer ses opinions, il paraît nécessaire de définir plus clairement les limites du pouvoir des employeurs, en tenant compte de ces lacunes.

L'existence d'une politique interdisant certains comportements sur les réseaux sociaux en dehors des heures de travail ne devrait pas suffire à justifier un licenciement lorsque ces comportements se produisent.

La gravité des comportements en question devrait être systématiquement examinée et il conviendrait de définir plus précisément l'étendue du pouvoir de contrôle de l'employeur sur les communications sans rapport avec l'entreprise et en dehors des heures de travail. 